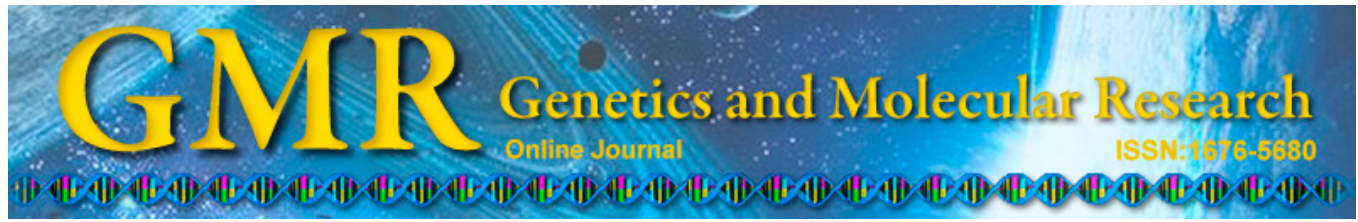

\title{
Relationship between polymorphism of SOCS- 3 and dyslipidemia in China Xinjiang Uygur
}

\author{
X.G. Yao, J. Meng, L. Zhou, N. Lin, J. Hong, M. Heizhati and N.F. Li \\ Hypertension Institute of Xinjiang China, \\ Hypertension Center of the People's Hospital of Xinjiang Uygur Autonomous \\ Region, Urumqi, Xinjiang, China \\ Corresponding author: N.F. Li \\ E-mail: lnanfang2010@sina.com.cn
}

Genet. Mol. Res. 14 (1): 1338-1346 (2015)

Received November 22, 2013

Accepted March 26, 2014

Published February 13, 2015

DOI http://dx.doi.org/10.4238/2015.February.13.13

\begin{abstract}
We investigated the relationship between the polymorphism of SOCS-3 and dyslipidemia of people from Uygur in Xinjiang, China. This cross-sectional study included 1379 participants in a Hetian Xinjiang Uygur population who were 30-70 years of age and were not from interracial marriages of 3 generations; all subjects were genotyped (909 dyslipidemia subjects, 470 healthy subjects). Allele $(\mathrm{P}=0.002)$ and genotype $(\mathrm{P}=0.003)$ frequencies of the distribution of rs12953258 was significantly different between dyslipidemia and control groups. Between the total cholesterol abnormal and control groups, high-density lipoprotein cholesterol abnormal and control groups, triglycerides abnormal and control groups, the frequencies of genotype in rs 12953258 were significantly different $(\mathrm{P}=0.007$, $0.012,0.0004$, respectively). Based on the logistic regression analysis, genotype CA and AA of rs12953258 were independent and risk factors for dyslipidemia in Uygur $(\mathrm{CC} v s \mathrm{CA}$; odds ratio $=1.48,95 \%$ confidence interval $=1.11-1.98, \mathrm{P}=0.008),(\mathrm{CC} v s \mathrm{AA}$; odds ratio $=2.48,95 \%$ confidence interval1.07-5.79, $\mathrm{P}=0.035$ ). Genotype AA of rs12953258 merged with subjects whose waist-to-hip ratio was abnormal, indicating the presence of dyslipidemia. The frequency of haplotype 4(H4) A-G-C
\end{abstract}


in the dyslipidemia group was higher than in the control group (8.44 vs $5.37 \%, \mathrm{P}=0.003)$. rs 12953258 site of the SOCS-3 gene showed a close relationship with dyslipidemia in Uygur. Combining genotype AA with subjects whose waist-to-hip ratios were abnormal will increase prevalence of dyslipidemia obviously.

Key words: Dyslipidemia; Metabolic syndrome; SOCS-3 gene

\section{INTRODUCTION}

Dyslipidemia, a metabolic syndrome (MS) (Alberti et al., 2005; Nanfang et al., 2010), is related to insulin resistance and obesity, which are risk factors of cardiovascular disease (Stem, 1999; Cabrera et al., 2012) and affected by genetics and the environment. A large number of studies have indicated that obesity, which is caused by leptin resistance, is a chronic low-grade metabolic inflammation. Obese patients who exhibit leptin resistance or mutations in the leptin receptor gene likely have altered lipid metabolism. Because insulin resistance and leptin resistance interact synergistically, these factors may lipid metabolism in the entire body.

Previous studies have supported that suppressor of cytokine signaling-3 (SOCS-3), which is located on the human chromosome $17 \mathrm{q} 25.3$, is a cytokine that signals to feedback inhibitors in the Janus kinase signal transducer and activator of transcription pathway (Endo et al., 1997; Qin et al., 2012). SOCS-3 participates in the development of many diseases, such as insulin resistance (Jorqensen et al., 2013), leptin resistance (Gu et al., 2009), immunopathy, inflammatory disease, and cancer (Lin et al., 2012). Thus, the alterations in the SOCS-3 gene may result in dyslipidemia.

The Uygur people from the Xinjiang minority area shows a prevalence rate of dyslipidemia as high as $34.8 \%$ is the high-risk group (Yao et al., 2010). This group has a relatively closed social environment, special diet, such as high-salt diet, high-fat food, and low-dietary fiber diet, and a very low interracial marriage rate. Thus, Uygur from the Xinjiang minority can be examined in genetic studies. Few studies have examined the relationship between SOCS-3 polymorphisms and dyslipidemia in humans. In this study, we investigated the relationship between SOCS-3 polymorphisms and dyslipidemia.

\section{MATERIAL AND METHODS}

\section{Participants}

In this cross-sectional epidemiological study, from January to March 2010, we randomly selected 1379 participants of Uygur from Hetian, a city in South Xinjiang. These participants included 521 males $(37.78 \%)$ and 858 females $(62.22 \%)$ who were $30-70$ years of age, with an average age of $51.14 \pm 11.22$, and no interracial marriage for 3 generations. Participants with thyroid disease, serious liver and kidney disease, and cancer were excluded. This study was approved by the local Ethics Committee, Health Bureau of Xinjiang Uygur Autonomous Region and People's Hospital of Xinjiang Uygur Autonomous Region. All participants signed informed consent. 


\section{Collection and standard of diagnosis}

Through cluster random sampling, participants were examined by questionnaire, physical examination, and biochemical index examination. Baseline data included name, gender, age, alcohol-intake, and smoking history. Physical examination includes height, weight, waistline, hipline, pulse, systolic blood pressure (SBP), and diastolic blood pressure (DBP). Additionally, body mass index (BMI) was calculated as weight/height ${ }^{2}$. According to prevention and control guidelines of China's adult overweight and obesity (Working Group on "Obesity of China, 2004), $18.5 \mathrm{~kg} / \mathrm{m}^{2} \leq \mathrm{BMI}<24 \mathrm{~kg} / \mathrm{m}^{2}$ is defined normal, $24 \mathrm{~kg} / \mathrm{m}^{2} \leq \mathrm{BMI}<28 \mathrm{~kg} / \mathrm{m}^{2}$ is defined overweight, and BMI $\geq 28 \mathrm{~kg} / \mathrm{m}^{2}$ is defined as obese. Mean arterial pressure, normal value of which ranges from 70-105 mmHg, was calculated as 1/3 (SBP + 2.DBP). The waist-tohip ratio (WHR) was calculated by dividing the waistline by the hipline (male $<0.90$, female $<$ 0.85 is defined normal). According to the China Adult Dyslipidemia Prevention Guide in 2007 (Joint Committee of Chinese Adult Dyslipidemia Prevention Guideline, 2007), dyslipidemia is defined as total cholesterol (TC) $\geq 6.22 \mathrm{mM}$, low-density lipoprotein cholesterol (LDL-C) $\geq 4.14$ $\mathrm{mM}$, serum triglyceride $(\mathrm{TG}) \geq 2.26 \mathrm{mM}$, high-density lipoprotein cholesterol (HDL-C) $<1.04$ $\mathrm{mM}$, or by the presence of 1 or more abnormal serum lipid concentration. If participants did not meet the above requirements, they were included in the control group.

\section{Biochemical index examination}

All participants ate a vegetarian diet with temperance for 3 days, and ate and drank water prohibitively for $12 \mathrm{~h}$. In the morning of the 4th day, the fasting elbow vein blood was collected. Blood samples were centrifuged at $944 \mathrm{~g}$ for $30 \mathrm{~min}$, plasma and blood cells were separated, and the samples were stored at $-20^{\circ} \mathrm{C}$. Biochemical indices included fasting blood glucose (FBG), TC, HDL-C, LDL-C, and TG. All biochemical indices were measured in the clinical laboratory of the People's Hospital of Xinjiang Uygur Autonomous Region.

\section{SOCS-3 DNA extraction and genotyping}

First, DNA from $200 \mu \mathrm{L}$ blood cells was extracted using the Paxgene blood DNA kit, AQIAGEN/BD Biosciences, Franklin Lakes, NJ, USA (PAXgene ${ }^{\mathrm{TM}}$ ) and then stored at $-80^{\circ} \mathrm{C}$. Based on relevant literature and software, 3 single nucleotide polymorphisms (SNPs) were selected: -920C/A (rs12953258) (Holter et al., 2007), 930A/G (rs4969168) (Fischer-Rosinsky et al., 2008), -6732C/T (rs9914220) (Talbert et al., 2009). All subjects were genotyped using TaqMan-polymerase chain reaction "with an 7900 REAL-TIME PCR apparatus (Applied Biosystems, Foster City, CA, USA). For quality control during genotyping, each 384-well plate was designed to include blank controls and positive controls, and case-control samples were randomly distributed on the plate. The call rate for genotyping was $98.9 \%$. The concordance of duplicates was $100 \%$.

\section{Statistical analysis}

The SPSS 17.0 statistical software (SPSS, Inc., Chicago, IL, USA) was used for analysis and continuous variables used to confirm a normal distribution are reported as means \pm standard deviation; variables showing non-normal distribution are reported as the median and 
interquartile range. Continuous variables were examined using the $t$-test, the Kruskal-Wallis $\mathrm{H}$-test, or analysis of variance. Categorical data of between-group design were examined using the $\chi^{2}$ test. Logistic regression analysis was performed for exposure risk factors of lipid metabolic disorder. All tests were two-tailed and the significance level was set at $\mathrm{a}=0.05$. P $<0.05$ indicated statistical significance. Hardy-Weinberg equilibrium was examined using the SNP Alyze7.0 software (DYNACOM Co., Ltd., Mobara, Japan).

\section{RESULTS}

\section{General characteristics}

In this study, the number of male and female subjects in the dyslipidemia group was higher than that in the control group. However, age in the dyslipidemia group was lower than that in the control group $(\mathrm{P}<0.05)$. For different lipid compositions, TC and HDL-C in the dyslipidemia group were all lower than those in the control group, and TG in the dyslipidemia group was higher than that in the control group $(\mathrm{P}<0.05)$. The difference in LDL-C in dyslipidemia and control groups was not statistically significant. For obesity-related indices, $\mathrm{BMI}$ and WHR in the dyslipidemia group were higher than those in the control group $(\mathrm{P}<$ 0.05). Mean arterial pressure, SBP, and DBP in dyslipidemia and control groups showed no significant difference. FBG in the dyslipidemia group was lower than that in the control group $(\mathrm{P}<0.05$; Table 1$)$.

Table 1. General characteristics between lipid metabolic disorder and control groups ( $\mathrm{N}=1379)$.

\begin{tabular}{lccr}
\hline & Case group $(\mathrm{N}=909)$ & Control group $(\mathrm{N}=470)$ & \multicolumn{1}{c}{$P$} \\
\hline Male/female & $364 / 545$ & $157 / 313$ & 0.016 \\
Age & $50.51 \pm 10.96$ & $52.34 \pm 11.63$ & 0.003 \\
TC $(\mathrm{mM})$ & $4.45 \pm 1.53$ & $4.47 \pm 0.81$ & 0.007 \\
HDL-C $(\mathrm{mM})$ & $0.96 \pm 0.35$ & $1.35 \pm 0.28$ & $<0.001$ \\
LDL-C $(\mathrm{mM})$ & $2.60 \pm 1.32$ & $2.51 \pm 0.79$ & 0.106 \\
TG $(\mathrm{mM})$ & $1.49(0.98,2.31)$ & $1.11(0.86,1.43)$ & $<0.001$ \\
BMI $\left(\mathrm{kg} / \mathrm{m}^{2}\right)$ & $27.54 \pm 4.48$ & $25.99 \pm 4.59$ & $<0.001$ \\
WHR & $0.91 \pm 0.17$ & $0.88 \pm 0.09$ & $<0.001$ \\
MAP $(\mathrm{mmHg})$ & $96.12 \pm 17.15$ & $96.21 \pm 18.87$ & 0.566 \\
SBP $(\mathrm{mmHg})$ & $130.45 \pm 25.35$ & $131.58 \pm 27.17$ & 0.657 \\
DBP $(\mathrm{mmHg})$ & $78.95 \pm 14.67$ & $78.53 \pm 16.52$ & 0.263 \\
FBG $(\mathrm{mM})$ & $5.13(4.49,6.05)$ & $5.33(4.87,6.09)$ & $<0.001$ \\
\hline Contim & & & \\
\hline
\end{tabular}

Continuous variables showing normal distribution are reported as means \pm standard deviation; non-normal data are reported as the median and interquartile range. Between case and control groups gender, age, body mass index (BMI), waist-to-hip ratio (WHR), fasting blood glucose (FBG), total cholesterol (TC), high-density lipoprotein cholesterol (HDL-C), and serum triglyceride $(\mathrm{TG})$ showed significant differences $(\mathrm{P}<0.05)$.

\section{Genotype frequency distribution of SOCS-3 in dyslipidemia and control groups}

The 3 gene mutation sites in the SOCS-3 gene (rs12953258, rs4969168, rs9914220) were in accordance with Hardy-Weinberg equilibrium. Between dyslipidemia and control groups, the frequency of genotype distribution $(\mathrm{P}=0.003)$ and allelic $(0.002)$ frequencies in rs12953258 showed a significant difference. Allele A of rs12953258 frequency in the case group (15.29\%) was higher than that in the control group (10.43\%). Between these 2 groups, neither rs4969168 nor rs9914220 showed a significant difference in the distribution of geno- 
type and allelic frequencies $(\mathrm{P}>0.05$; Table 2$)$.

Table 2. Genotype frequency distribution of SOCS-3 in dyslipidemia and control groups.

\begin{tabular}{|c|c|c|c|c|c|}
\hline SNPs & Allele or genotype & Case group (\%) & Control group (\%) & $\chi^{2}$ & $P$ \\
\hline \multirow[t]{5}{*}{ rs4969168 } & GG & $387(42.60)$ & $175(37.20)$ & 4.8 & 0.09 \\
\hline & GA & $402(44.30)$ & $237(50.30)$ & & \\
\hline & AA & $119(13.10)$ & $59(12.50)$ & & \\
\hline & $\mathrm{G}$ & $1176(64.76)$ & $587(62.31)$ & 1.606 & 0.21 \\
\hline & $\mathrm{A}$ & $640(35.24)$ & $355(37.69)$ & & \\
\hline \multirow[t]{5}{*}{ rs9914220 } & TT & $43(4.80)$ & $20(4.20)$ & 2.12 & 0.35 \\
\hline & $\mathrm{CT}$ & $305(33.70)$ & $143(30.20)$ & & \\
\hline & $\mathrm{CC}$ & $557(61.50)$ & $310(65.50)$ & & \\
\hline & $\mathrm{T}$ & $391(21.60)$ & $183(19.34)$ & 1.92 & 0.17 \\
\hline & $\mathrm{C}$ & $1419(78.40)$ & $763(80.66)$ & & \\
\hline \multirow[t]{5}{*}{ rs12953258 } & $\mathrm{CC}$ & $661(72.72)$ & $379(80.64)$ & 11.65 & 0.003 \\
\hline & $\mathrm{CA}$ & $218(23.98)$ & $84(17.87)$ & & \\
\hline & AA & $30(3.30)$ & $7(1.50)$ & & \\
\hline & $\mathrm{C}$ & $1540(84.71)$ & $842(89.57)$ & 12.46 & $<0.001$ \\
\hline & A & $278(15.29)$ & $98(10.43)$ & & \\
\hline
\end{tabular}

\section{Genotype frequency distribution of rs12953258 with different lipid composition}

The distribution of different genotypes in rs12953258 was different between abnormal TC and control groups $(\mathrm{P}=0.007)$, abnormal HDL-C and control groups $(\mathrm{P}=0.012)$, and abnormal TG and control groups $(\mathrm{P}=0.0004)$. For the genotypic frequency distribution in rs12953258, we observed no clear difference between abnormal LDL-C and control groups $(\mathrm{P}>0.05$; Figure 1).
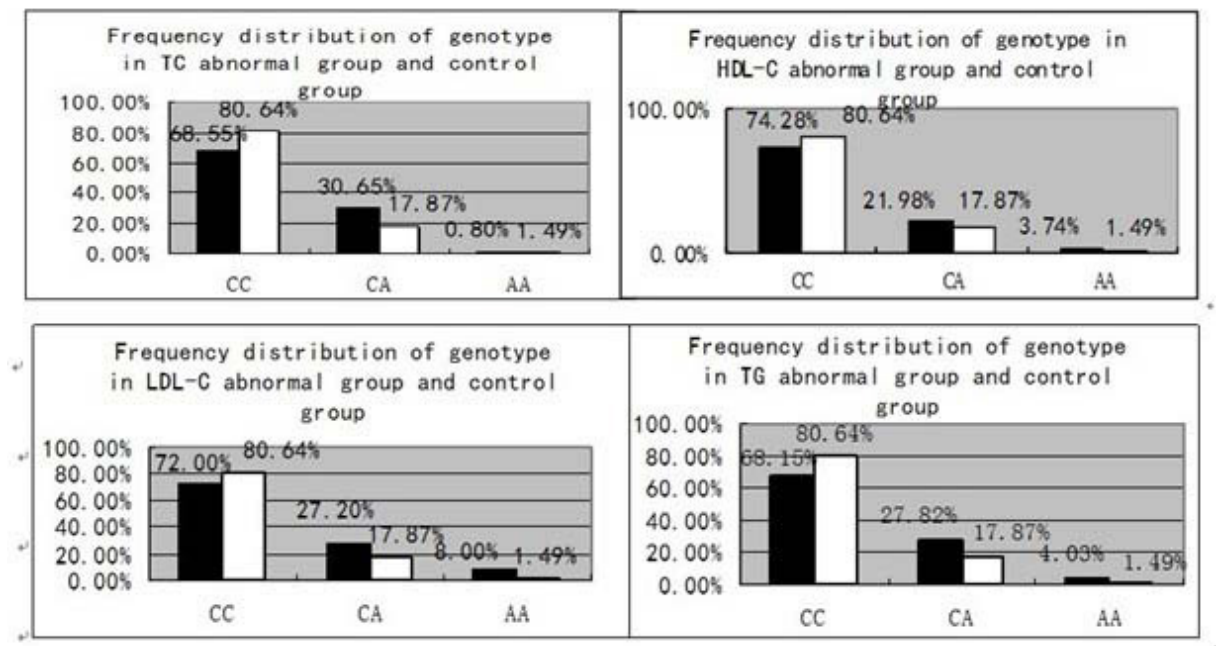

Figure 1. Three genotype frequencies of rs 12953258 in different lipid component. Filled columns $=$ abnormal groups. Open columns = lipid metabolism of normal groups. Three genotype's frenquency distribution of rs12953258 have statistically significant difference in the TC abnormal group and lipid metabolism of normal group $\left(\chi^{2}=10.00, \mathrm{P}=0.007\right)$, HDL-C abnormal group and lipid metabolism of normal group $\left(\chi^{2}=8.81, \mathrm{P}=0.012\right)$, TG abnormal group and lipid metabolism of normal group $\left(\chi^{2}=15.3, \mathrm{P}=0.0004\right)$, respectively. Unfortunately, we cannot find in the three genotype's frenquency distribution of rs13953258 any significant difference in HDL-C abnormal group and lipid metabolism of normal group $\left(\chi^{2}=3.793, \mathrm{P}=0.15\right)$. 


\section{Logistic regression analysis}

Based on logistic regression analysis, the lipid metabolic condition was the dependent variable. The independent variables were rs12953258, rs4969168, rs9914220, age, gender, and BMI, WHR normal, mean arterial pressure, SBP, DBP, and FBG. Between dyslipidemia and control groups, the genotypes CA and AA of rs12953258 were independent risk factors for dyslipidemia in Uygur from the minority area of Xin Jiang [CC vs CA; odds ratio (OR) = $1.48,95 \%$ confidence interval $(\mathrm{CI})=1.11-1.98, \mathrm{P}=0.008],(\mathrm{CC}$ vs $\mathrm{AA} ; \mathrm{OR}=2.48,95 \% \mathrm{CI}$ $=1.07-5.79, \mathrm{P}=0.035)$. Genotype $\mathrm{CC}$ is a protective factor in lipid metabolism (AA vs CC, $\mathrm{OR}=0.41,95 \% \mathrm{CI}=0.18-0.94, \mathrm{P}=0.034)$. BMI, WHR, and being male are risk factors for dyslipidemia. Combining genotype AA with abnormal WHR resulted in a higher prevalence of dyslipidemia than genotype $\mathrm{CC}$ with normal WHR $(\mathrm{OR}=6.17,95 \% \mathrm{CI}=1.40-27.11, \mathrm{P}=$ 0.016). In females, the genotype AA showed a higher prevalence of dyslipidemia than genotype $\mathrm{CC}(\mathrm{OR}=3.98,95 \% \mathrm{CI}=1.46-10.85, \mathrm{P}=0.007$; Table 3$)$.

\begin{tabular}{|c|c|c|c|c|c|}
\hline Independent variable & B & SE & Wales & OR $(95 \% \mathrm{CI})$ & $\mathrm{P}$ \\
\hline \multicolumn{6}{|l|}{ rs12953258 } \\
\hline $\mathrm{CC}$ & - & - & 11.39 & & 0.003 \\
\hline $\mathrm{CA}$ & 0.39 & 0.15 & 7.08 & $1.48(1.11-1.98)$ & 0.008 \\
\hline AA & 0.97 & 0.43 & 5.09 & $2.64(1.14-6.14)$ & 0.024 \\
\hline Age & -0.16 & 0.01 & 9.26 & $0.98(0.97-0.99)$ & 0.002 \\
\hline Gender & 0.3 & 0.12 & 5.8 & $1.35(1.06-1.72)$ & 0.016 \\
\hline BMI & 0.05 & 0.02 & 11.93 & $1.05(1.02-1.08)$ & 0.001 \\
\hline Normal WHR or not & 0.53 & 0.13 & 17 & $1.70(1.32-2.19)$ & $<0.001$ \\
\hline $\mathrm{CC}+$ normal WHR & - & - & 27.33 & 1.0 & $<0.001$ \\
\hline $\mathrm{CC}+$ abnormal WHR & 0.62 & 0.13 & 22.35 & $1.86(1.44-2.40)$ & $<0.001$ \\
\hline AA + abnormal WHR & 1.82 & 0.76 & 5.81 & $6.17(1.40-27.11)$ & 0.016 \\
\hline $\mathrm{AA}+$ normal WHR & - & - & 27.33 & 1.0 & $<0.001$ \\
\hline $\mathrm{AA}+$ abnormal WHR & 1.05 & 0.91 & 1.32 & $2.86(0.48-17.11)$ & 0.25 \\
\hline Female $+\mathrm{CC}$ & - & - & 10.32 & 1.0 & 0.017 \\
\hline Female + AA & 1.38 & 0.51 & 7.29 & $3.98(1.46-10.85)$ & 0.007 \\
\hline
\end{tabular}

Lipid metabolic condition was the dependent variable. rs4969168 and rs9914220 were independent variables. Unfortunately, we did not notice that rs4969168 and rs9914220 have relationship with dyslipidemia in Uygur $(\mathrm{P}>0.05)$.

\section{Distribution of haplotype frequency in dyslipidemia and control groups}

In this general population, the frequency of haplotype $4(\mathrm{H} 4)$ A-G-C in the dyslipidemia group was higher than that in the control group ( 8.44 vs $5.37 \%, \mathrm{P}=0.003)$. Haplotype 6 (H6) A-A-C, haplotype 7 (H7) A-G-T, and haplotype 8 (H8) A-A-T were not listed, because their frequency $<5 \%$ in the general population (Table 4 ).

Table 4. Distribution of haplotype frequency in dyslipidemia and control groups.
\begin{tabular}{lcccc}
\hline Haplotype & rs12953258/rs4969168/rs9914220 & Dyslipidemia group [N, (\%)] & Control group [N, (\%)] & P \\
\hline H1 & C-G-C & $392(43.16)$ & $215(45.69)$ & 0.209 \\
H2 & C-A-C & $213(23.38)$ & $125(26.63)$ & 0.064 \\
H3 & C-G-T & $99(10.84)$ & $48(10.18)$ & 0.622 \\
H4 & A-G-C & $77(8.44)$ & $25(5.37)$ & 0.003 \\
H5 & C-A-T & $68(7.46)$ & $33(7.08)$ & 0.826 \\
\hline
\end{tabular}

The haplotype which frequency $<5 \%$ is not listed. 


\section{DISCUSSION}

The SOCS-3 gene located on the human chromosome 17q25.3 is a significant member of the SOCS gene family. SOCS-3 participates in the signal transduction inhibiting factor of Janus kinase signal transducer and activator of transcription pathway, which is an important method for the body to adjust its immune responses, inflammation, and had interrelationship with other pathways (Kisseleva et al., 2002). A large number of studies have argued that the SOCS-C gene has effects in many diseases. Through its interaction with the insulin receptor, the SOCS-3 gene gives rise to insulin resistance, which can induce high serum TG and HDLC (Zhang and Yang, 2003). Insulin resistance is the link to the relationship between SOCS-3 gene polymorphisms, and dyslipidemia provided the theoretical basis for this study.

There are 3 gene mutation sites in the SOCS-3 gene, including rs 12953258, rs4969168, and rs9914220) that were applicable to this study. rs12953258, located in the 5'-untranslated region area, is closely related to dyslipidemia in Uygur from the minority area of Xinjiang. The genotype AA and allele A are likely risk factors for dyslipidemia. Additionally, haplotypes that are variables of linkage areas show more polymorphisms than SNP. Haplotype analysis revealed that $\mathrm{H} 4 \mathrm{~A}-\mathrm{G}-\mathrm{C}$ was related to dyslipidemia.

The study also showed that the SOCS-3 gene rs12953258 was closely related to TC, HDL-C, and TG. However, we observed no relationship between rs12953258 and LDL-C. This may be explained by many factors: 1) the 3 selected sites of gene mutation failed to represent or explain other sites of the SOCS-3 gene, which may affect the level of LDL-C. A few sites of low-frequency mutations must be researched and analyzed. 2) Different races have different genetic characteristics, social environment, and diet. Additional experiments are necessary that include subjects of many races. However, we found that age, TC, HDL-C, LDL-C, TG, BMI, and WHR between different genotypes of rs 12953258 showed no statistical significant difference $(\mathrm{P}>0.05)$. Two likely explanations are: 1$)$ SOCS-3 may be a minor gene and 2) a relationship may exist with fewer cases of genotype AA.

WHR, BMI, and being male may be risk factors for dyslipidemia. The number of male subjects with dyslipidemia was 1.341 times higher than that of female subjects with dyslipidemia. This may be because both external and endogenous estrogen can reduce the accumulation of LDL-C in arterial walls, which may reduce the concentration of lipoprotein in blood circulation (Gotto Jr., 1994) while increasing HDL-C (Matusubara et al., 1997). Most previous studies only reported the prevalence of dyslipidemia in males and females; however, the prevalence ratio between males and females has not been thoroughly reported, particularly in minority areas of Xinjiang. Thus, this ratio may be a reference for studies of lipid metabolism in the future. Additionally, for rs 12953258, dyslipidemia prevalence in subjects with the genotype AA was 2.64 times higher than that in subjects with the genotype CC. Dyslipidemia prevalence of those with genotype AA and abnormal WHR was 6.17 times higher than that of subjects with the genotype CC and normal WHR. Thus, SOCS-3 is a susceptibility gene. Both environment and heredity affect dyslipidemia. In subjects with genotype AA, an abnormal WHR resulted in a prevalence of dyslipidemia that was 2.86 times higher than that in subjects with normal WHR values, but $\mathrm{P}>0.05$. This may have been because of the low proportion of genotype AA cases ( 37 subjects; $2.7 \%$ ). Thus, additional studies of a larger population including different races are necessary. 


\section{CONCLUSION}

In this study, we identified a close relationship between the SOCS-3 gene and dyslipidemia, which can be affected by the environment and heredity factors. Based on our results, a large study to examine lipid metabolism and the relationship with rs 12953258 in the SOCS-3 gene, particularly focusing on subjects with the genotype AA and an abnormal WHR, primary methods for preventing diseases to reduce the prevalence of dyslipidemia can be developed.

\section{ACKNOWLEDGMENTS}

We would like to thank both the subjects of study to participate in the present research and the staff of Diagnosis, Treatment and Research of Hypertension in Xinjiang. Research supported by the National Natural Sciences Foundation of China (\#31060157).

\section{REFERENCES}

Alberti KG, Zimmet P, Shaw J and IDE Epidemiology Task Force Consensus Group (2005). The metabolic syndrome - a new worldwide definition. Lancet 366: 1059-1062.

Cabrera MA, de Andrade SM and Mesas AE (2012). A prospective study of risk factors for cardiovascular events among the elderly. Clin. Interv. Aging 7: 463-468.

Endo TA, Masuhara M, Yokouchi M, Salamoto H, et al. (1997). A new protein containing an SH2 domain that inhibits JAK kinases. Nature 387: 921-924.

Fischer-Rosinsky A, Fisher E, Kovacs P, Blüher M, et al. (2008). Lack of association between the tagging SNP A+930->G of SOCS3 and type 2 diabetes mellitus: meta-analysis of four independent study populations. PloS One 3: e3853.

Gotto AM Jr (1994). Postmenopausal hormone-replacement therapy, plasma lipoprotein[a], and risk for coronary heart disease. J. Lab. Clin. Med. 123: 800-803.

$\mathrm{Gu}$ H, Liu L, Ma S, Liu Y, et al. (2009). Inhibition of SOCS-3 in adipocytes of rats with diet-induced obesity increase leptinmediated fatty acid oxidation. Endocrine 36: 546-554.

Holter K, Wermter AK, Scherag A, Siegfried W, et al. (2007). Analysis of sequence variations in the suppressor of cytokine signaling (SOCS-3) gene in extremely obese children and adolescents. BMC Med. Genet. 8: 1-6.

Joint Committee for Developing Chinese Guidelines on Prevention and Treatment of Dyslipidemia in Adults (2007). Chinese adult dyslipidemia prevention guidelines. Chin. J. Cardiol. 35: 390-419.

Jorqensen SB, O’Neil HM, Sylow L, Honeyman J, et al. (2013). Deletion of skeletal muscle SOCS-3 prevents insulin resistance in obesity. Diabetes 61: 56-64.

Kisseleva T, Bhattacharya S, Braunstein J and Schindler CW (2002). Signaling through the JAK/STAT pathway, recent advances and future challenges. Gene 285: 1-24.

Lin N, Yao XG and Li NF (2012). Research advances in suppressor of cytokine signaling 3. Chin. J. Acta Acad. Med. Sci. 34: $178-182$.

Matusubara Y, Murata M, Kawano K, Zama T, et al. (1997). Genotype distribution of estrogen receptor polymorphisms in men and postmenopausal woman from healthy and coronary populations and its relation to serum lipid levels. Arterioscler. Thromb. Vasc. Biol. 17: 3006-3012.

Nanfang L, Yanying G, Hongmei W, Zhitao Y, et al. (2010). Variations of six transmembrane epithelial antigen of prostate 4 (STEAP4) gene are associated with metabolic syndrome in a female Uygur general population. Arch. Med. Res. 41: 449-456.

Qin H, Holdbrooks AT, Liu Y, Reynolds SL, et al. (2012). SOCS-3 deficiency promotes M1 macrophage polarization and inflammation. J. Immunol. 189: 3439-3448.

Stem MP (1999). Natural history of macrovascular disease in type 2 diabetes. Diabetes Care 22: c2-5.

Talbert ME, Langefeld CD, Ziegler J, Mychaleckyj JC, et al. (2009). Polymorphisms near SOCS3 are associated with obesity and glucose homeostasis traits in Hispanic Americans from the insulin resistance atherosclerosis family study. Hum Genet. 125: 153-162.

Working Group on Obesity of China (WGOC) (2004). Prevention and control guidelines of Chinese adult overweight and 
obesity. Chin. Newspaper Acta Nutrimenta Sin. 26.

Yao XG, Frommlet F, Zhou L, Zu F, et al. (2010). The prevalence of hypertension, obesity and dyslipidemia in individuals of over 30 years of age belonging to minorities from the pasture area of Xinjiang. BMC Public Health 10: 91.

Zhang Y and Yang XJ (2003). Relationship between lipid metabolism disorders in patients with type 2 diabetes and coronary heart disease (CHD). Chin. J. Clin. Int. Med. 20: 634-635. 